\title{
Opioid Misuse and Neural Responses to Social Rejection
}

\author{
THIS IS AN UNPUBLISHED PREPRINT AND HAS NOT BEEN ACCEPTED AFTER \\ PEER REVIEW AT ANY OUTLET. PLEASE DO NOT CITE THIS MANUSCRIPT \\ WITHOUT THE AUTHORS' PERMISSION.
}

David S. Chester ${ }^{1 *}$, Sarah Beth Bell ${ }^{2}$, Olivia Vanegas ${ }^{3}$, William W. Stoops ${ }^{4}$, C. Nathan DeWall ${ }^{5}$

\author{
${ }^{1}$ Department of Psychology, Virginia Commonwealth University, USA \\ ${ }^{2}$ School of Community Medicine, University of Oklahoma, USA \\ ${ }^{3}$ Department of Psychology, University of Connecticut, USA \\ ${ }^{4}$ Department of Behavioral Science, University of Kentucky, USA \\ ${ }^{5}$ Department of Psychology, University of Kentucky, USA
}

${ }^{*}$ Correspondence should be addressed to:

David S. Chester

Department of Psychology

Virginia Commonwealth University

Richmond, VA, 23284, USA

$1-804-828-7624$

dschester@vcu.edu 


\begin{abstract}
Opioid misuse is a costly public health issue that often develops in response to physical pain. However, the roles of social pain in opioid misuse and the neural mechanisms that convey its potential effects are largely uninvestigated. Twenty eight participants who reported recreational opioid misuse in the past 30 days completed a study in which they were socially accepted and then rejected while undergoing functional magnetic resonance imaging (fMRI). Participants who misused opioids displayed a typical pattern of neural reactivity to rejection across broad swaths of cortical and subcortical regions, though subjective reports of rejection-related threat were encoded in brain regions with dense concentrations of opioid receptors. Greater post-rejection cravings for opioids were linked to a deficit in ventromedial prefrontal cortex (VMPFC) activity during social acceptance. Compared to healthy controls, participants who misused opioids exhibited greater VMPFC activity during rejection. These findings suggest that people who misuse opioids have intact neural processing of rejection events and that the distress of these events may be contributed to by dysfunction in the opioid system. Further, opioid cravings may arise when these individuals do not adequately pair social acceptance with a neural 'safety signal'.
\end{abstract}

Keywords: opioid misuse, opioid dependence, social rejection, fMRI, pain 


\section{Introduction}

Opioids blunt the aversive experience of pain and influence many social psychological processes (Panksepp, Herman, Conner, Bishop, \& Scott, 1978). Many individuals misuse opioids, by taking them recreationally or in greater amounts or frequencies than is medically prescribed. In 2018 alone, approximately 10.3 million people in the United States (i.e., 3.7\% of the U.S. population) misused opioids and 2.0 million people had an opioid use disorder ( $0.7 \%$ of the U.S. population; National Survey on Drug Use and Health, 2019). To examine how pain contributes to opioid misuse, research has almost exclusively focused on physical pain. In what follows, we describe an experiment that employed functional neuroimaging to examine whether people who misuse opioids exhibited altered brain responses to social pain, and whether this contributed to their subsequent craving for opioids.

\section{Mechanisms and Functions of Opioids}

Pain is a critically-important experience to detect and remedy injuries, yet it is also one that leads to profound impairment. To function optimally, humans have evolved endogenous opioid systems to help manage the experience of pain. Internallygenerated neurotransmitters, such as endorphins, bind to opioid receptors in circumscribed regions of the brain, primarily the thalamus, striatum, amygdala, hippocampus, and adjoining cortical regions such as the ventromedial prefrontal cortex (Nummenmaa \& Tuominen, 2018). Opioid binding at these receptors affects neural activity in ways that attenuate the aversive experience of pain and increases the subjective experience of pleasure - which can contribute to misuse of exogenously administered opioid drugs (Contet, Kieffer, \& Befort, 2004). Misuse of exogenous 
sources of opioids (e.g., prescription pain medicines) can have costly, long-term effects.

\section{Opioid Misuse and Pain}

Opioid misuse occurs when individuals use opioids outside of a legitimate medical prescriptions (e.g., recreational use, taking larger doses than is prescribed, taking more frequent doses than is prescribed). Although not all misuse results in development of opioid use disorder, opioid misuse can lead to substantial problems, including opioid use disorder and physical dependence. Research into the causes and risk factors of opioid misuse have largely focused on physical pain. Indeed, chronic physical pain conditions and pain disorders are reliable risk factors for opioid misuse (Voon, Karamouzian, \& Kerr, 2017). However, pain is not purely physical.

\section{Social Pain}

Physical injuries promote the experience of physical pain. In a parallel fashion, social injuries (e.g., rejection, ostracism, exclusion, isolation, stigma, provocation, marginalization) can cause the aversive experience of social pain (Eisenberger, 2012; Eisenberger \& Lieberman, 2004; MacDonald \& Leary, 2005). Distress from social pain arises in the affective components of the brain's pain matrix (i.e., anterior insula, dorsal anterior cingulate cortex $[\mathrm{ACC}])$, often excluding the somatosensory components of this circuit (Eisenberger, 2012; Eisenberger, Lieberman, \& Williams, 2003; Rotge et al., 2014). In addition to recruiting neural pain circuitry, social pain is attenuated by analgesics (DeWall et al., 2010), heightened among individuals with greater physical pain sensitivity (Eisenberger, Jarcho, Lieberman, \& Naliboff, 2006), and is increased during inductions of physical pain (Riva, Wirth, \& Williams, 2011). This evidence suggests that social pain is a truly painful experience, and not merely a form of negative 
affect. Despite the strong evidence for social pain, it remains largely uninvestigated in its relation to opioid misuse.

\section{Reciprocal Effects of Opioid Functioning and Social Pain}

Yet why would social pain be involved in opioid misuse? A hint as to the existence of this potential link comes from evidence linking social pain to functioning of the brain's opioidergic circuitry. For instance, social rejection recruits the release and binding of endogenous opioids in multiple brain regions, ostensibly to blunt the experience of social pain (Hsu et al., 2013, 2015). Pharmacologically blocking opioid activity in the brain via opioid antagonists magnifies feelings of social pain among healthy adults (Inagaki, 2018; Inagaki, Irwin, \& Eisenberger, 2015; Inagaki, Ray, Irwin, Way, \& Eisenberger, 2016). Conversely, opioid agonists, such as buprenorphine, lessen feelings of social pain among healthy adults (Bershad, Seiden, \& de Wit, 2016). Across these forms of correlational and causal evidence, social pain appears to be modulated by opioids in a similar manner to physical pain.

\section{Altered Responses to Rejection Among People Who Misuse Opioids}

As further evidence for the intimate relationship between opioids and social pain, people who misuse opioids exhibit altered psychological and biological responses to social rejection. Compared to healthy controls, non-intoxicated people with histories of opioid misuse (including people with and without opioid dependence) also reported greater anger after an experience of social rejection but did not differ in their broader levels of negative and positive affect (Carlyle, Rowley, Stevens, Karl, \& Morgan, in press). The absence of group differences (between those who have and have not misused opioids) in negative and positive post-rejection affect was replicated in a 
separate sample of non-intoxicated people who misused opioids (including people with and without opioid dependence), though they did exhibit heightened rejection-related cortisol reactivity (Kroll et al., 2019). People with opioid dependence who were maintained on methadone also did not report greater rejection-related distress, though they did exhibit blunted ACC and occipital responses to rejection (Bach et al., 2019). Curiously, these participants with opioid dependence reported that they did not feel as included during social acceptance. This blunted inclusionary status was reflected in reduced occipital activity during social acceptance among participants who misused opioids (as compared to healthy controls). These studies suggest that those who misuse opioids do not experience rejection in a subjectively more distressing manner, but that their underlying neurobiological response to rejection is aberrant.

\section{The Present Study}

The existing research on neural responses to rejection and opioid misuse has focused on individuals with opioid dependence and who are undergoing treatment for this condition. Comparatively little research has evaluated neural responses to social rejection among those who misuse opioids recreationally and who are not undergoing treatment for their opioid misuse. Further, the relation between neural reactivity to rejection and subsequent cravings for opioids remains largely unexamined — rendering it unclear whether divergent neurobiological reactivity to exclusionary events may contribute to this crucial pathway underlying addiction. To fill these gaps in our knowledge, we sought to $(A)$ identify whether individuals who use opioids recreationally exhibited different patterns of neural reactivity to social acceptance and rejection than healthy controls, and to $(B)$ test potential links between these altered neural responses 
and subsequent opioid cravings. To do so, we experimentally-induced an experience of social acceptance then rejection among people who misused opioids while they underwent functional magnetic resonance imaging (fMRI) of the brain. After rejection, participants reported their current levels of subjective opioid craving and the extent to which they felt threatened by the rejection incident (a proxy for social pain). We compared these participants to a sex-matched sub-sample of an existing sample of controls who did not report opioid use that completed the same social rejection fMRI paradigm and self-reported rejection threat measure. No a priori predictions were made for this exploratory investigation.

\section{Open Practices Statement}

This study was not preregistered. De-identified data necessary to replicate our results are publicly-available here: https://osf.io/y3u2q/files/.

\section{Methods}

\section{Ethics Statement}

All of the following research procedures were approved by an institutional research ethics board, in accordance with institutional and national research ethics guidelines.

\section{Participants}

Opioid misuse sample. Potential participants were recruited from the general Lexington, KY community via flyers that advertised a study for 'people who have used opioids for non-medical reasons' and were 18-50 years old. Interested parties then completed a phone screen that required them to report at least one instance of opioid misuse (i.e., illicit misuse of opioids outside of a medically-prescribed regimen and for 
recreational purposes). Additional exclusionary criteria included in this phone screening were ages outside the range of 21 to 65 , experiencing withdrawal symptoms within eight hours of abstinence from opioids, lacking right-hand dominance, magnetic metal in the body, claustrophobia, colorblindness, current pregnancy, major medical conditions, history of traumatic brain injury, and current use of prescribed psychoactive medication. For their participation, participants received $\$ 65$ and images of their brain.

Participants in the opioid misuse sample were 28 adults who had self-reported that they had misused opioids over the past 30 days (gender: 10 females, 18 males; ethnicity: 1 Hispanic, 27 non-Hispanic; race: 2 Black, 26 White; see Table 1 for more information on this sample's characteristics). Indicating that our sample was mostly comprised of individuals with opioid dependence, a majority of participants reported that they experienced withdrawal during opioid abstinence $(N=21 ; 75.00 \%$ of sample). Participants misused opioids via intravenous injection ( $N=16 ; 57.15 \%$ of sample), intranasal administration $(N=5 ; 17.86 \%$ of sample), oral administration $(N=3 ; 10.71 \%$ of sample), a mixture of intranasal and oral administration ( $N=2 ; 7.14 \%$ of sample), or a mixture of intravenous injection and intranasal administration $(N=2 ; 7.14 \%$ of sample).

Table 1

Characteristics of participants who misused opioids.

\begin{tabular}{llll}
\hline Variable & $M$ & $S D$ & range \\
\hline Age & 37.14 & 7.53 & $24-50$ \\
Years of Education & 12.73 & 1.95 & $9-16$ \\
Monthly Income & $\$ 895.36$ & $\$ 791.54$ & $\$ 0-\$ 2,700$ \\
Age at First Opioid Misuse & 17.04 & 3.56 & $7-23$ \\
Days Since Last Opioid Misuse & 2.29 & 6.14 & $0-30$ \\
Days of Opioid Misuse in Past 30 Days & 22.18 & 8.95 & $2-30$ \\
Days of Marijuana Misuse in Past 30 Days & 5.39 & 7.69 & $0-29$ \\
Days of Alcohol Misuse in Past 30 Days & 3.96 & 8.47 & $0-29$ \\
Typical Opioid Dose & $178.89 \mathrm{mg}$ & $165.08 \mathrm{mg}$ & $5-500 \mathrm{mg}$ \\
\hline
\end{tabular}


Monthly Opioid Expenditure

$\$ 1,357.14 \quad \$ 1,768.80$

$\$ 0-\$ 9,000$

This sample size was determined by logistical constraints (i.e., available funds and recruitment success) and not by an a priori power analysis. Our sample size (i.e., $N$ $=28$ per group) provided $80 \%$ power to detect between-group main effects of $d=0.77$ and larger at $\alpha=.05$.

Control sample. Participants were 28 healthy adults ( 10 females, 18 males; age: $M=22.14, S D=3.02$, range: $18-30)$, who were acquired from an existing dataset ${ }^{1}$. These control participants were either undergraduates recruited through an introductory psychology subject pool at the University of Kentucky in exchange for credit towards their course's research requirement and images of their brain, or they were general Lexington, $\mathrm{KY}$ community members recruited in exchange for $\$ 50$ and images of their brain. Exclusionary criteria were ages outside the range of 18 to 35 , no right-hand dominance, metal in the body, claustrophobia, colorblindness, pregnancy, major medical conditions (e.g., psychopathologies, developmental disorders), history of traumatic brain injuries, current use of psychoactive medication, and body mass index above 30. Data were not acquired as to this group's opioid or other drug misuse, though they were required to have no history of such substance abuse.

\section{Materials}

Need Threat Scale. The 20 -item Need Threat Scale served as a manipulation check of the Cyberball paradigm's ability to elicit rejection threat (Riva et al., 2011; Van Beest \& Williams, 2006; Williams, 2009). This measure consisted of four, five-item subscales through which participants retrospectively reported the extent to which the

\footnotetext{
${ }^{1}$ Rejection-related fMRI and Need Threat Scale data from these participants, combined with participants from the full sample, has been previously-published (Chester \& DeWall, 2018; Chester, Lynam, Milich, \& DeWall, 2018).
} 
experience of rejection from the Cyberball task threatened participants' needs for belonging (sample item: "I felt like an outsider"), control (sample item: "I felt I had control over the game"), meaningful existence (sample item: "I felt meaningless), and selfesteem (sample item: "I felt liked"). Participants responded to each item along a 1 (not at all) to 5 (extremely) response scale among the opioid misuse sample, and a 1 (not at all) to 7 (extremely) response scale among the control sample. After we reverse-scored the appropriate items, we computed scores for each of the four subscales by averaging items together and then standardizing scores within sample so that both samples could be combined in later analyses.

Opioid craving measure. To measure post-rejection cravings for opioids, participants in the opioid misuse sample were verbally asked by an experimenter "on a scale from 1 (not at all) to 7 (extremely), how much do you want to take opioids right now?" (acquired from Garland, Bryan, Nakamura, Froeliger, \& Howard, 2017).

\section{Procedure}

Participants in the opioid misuse sample completed the following procedure (procedure for the control sample is detailed in Chester \& DeWall, 2018).

Pre-scan. After passing our phone screening, participants were scheduled to arrive at the University of Kentucky's Magnetic Resonance Imaging and Spectroscopy Center for a study that was ostensibly focused on opioid cravings and brain functioning during self-control tasks. Prior to arrival, participants were notified that they could administer opioids as close to the study session as they would need to $(A)$ not experience withdrawal symptoms during the session and (B) not be intoxicated during the session. Upon arrival to the lab, participants first completed a field sobriety test and 
then provided informed consent, reported on whether they had used alcohol or illicit drugs on the day of the study, and completed an MRI safety screening form. Participants also provided a urine sample that was tested for pregnancy (in females) and the presence of opioids, as well as other commonly misused drugs (i.e., stimulants and marijuana). Based on these test results, 22 participants from the opioid misuse sample tested positive for recent opioid use ( 5 for buprenorphine, 11 for fentanyl, 1 for methadone, 17 for morphine, 7 for oxycodone, 1 for tramadol), 10 tested positive for amphetamines, 4 tested positive for benzodiazepines, 4 tested positive for cocaine, 14 tested positive for marijuana, and 11 tested positive for methamphetamine. Such concurrent polydrug use is expected among participants who misuse opioids.

Participants who completed all of these procedures were instructed that they would be completing the study with two partners who were in nearby testing rooms. To ensure the believability of this deception, participants were told that they were the second participant to arrive and then they were asked to select from two remaining pieces of paper that would determine which of the three MRI scanners they would be placed in (in reality, there was only one MRI scanner). After being 'assigned' to their MRI scanner, the experimenter left the participant in the screening room to complete computer questionnaires while he/she went to 'see whether the other participants had arrived'. The experimenter then returned to the participant and asked them to complete a form that would give their partners some more information about them (e.g., hometown, favorite hobbies, favorite food). The experimenter then exchanged participants' information form with two pre-completed forms with sex-matched handwriting. After reading their partners' fictitious information, the experimenter 
collected the forms and walked participants through a computerized practice version of the Cyberball task, which only modeled social acceptance and not rejection.

MRI scan. Participants were then placed in an MRI scanner and had a highresolution structural scan taken of their brain. To induce an experience of social rejection in the functional neuroimaging environment, we employed the Cyberball social rejection manipulation (Chester et al., 2014, 2018; Eisenberger et al., 2003; Williams, Cheung, \& Choi, 2000). In this task, participants played a virtual ball-tossing game with two fictitious partners. Participants were informed that the purpose of the task was to mentally visualize the ball-tossing experience as if it were occurring in real life. The task contained three blocks. In the first two, 60-second blocks, participants received an equal number of ball-tosses from their two partners (Accept condition). However, in the third, 80-second block, participants stopped receiving the ball from their partners after 30 seconds who continuously threw it back-and-forth to one another for 50 seconds (Reject condition). Baseline neural activation was captured by 10 -second 'rest' trials that preceded each of the three blocks. Immediately after the Cyberball task and while still lying in the MRI scanner, participants verbally reported their current cravings for opioids. Participants then completed several other functional MRI tasks and were then removed from the scanner.

Post-scan. Participants were seated in an adjacent testing room and completed a battery of questionnaires that included the Need Threat Scale. Participants were then fully debriefed, paid, and dismissed.

\section{MRI Data Acquisition}

MRI data were obtained using a 3.0 Tesla Siemens MAGNETOM Prisma 
scanner (opioid misuse sample) or a 3.0 Tesla Siemens MAGNETOM Trio scanner (controls). Across all participants, echo planar imaging with a T2*-weighted gradient was applied across the entire brain with a 3D shim: matrix size $=64 \times 64$, field of view $=$ $224 \mathrm{~mm}$, echo time $(T E)=28 \mathrm{~ms}$, repetition time $(T R)=2.0 \mathrm{~s}, 3.5 \mathrm{~mm}^{3}$ voxel size, 40 interleaved axial slices, flip angle $=90^{\circ}$. To allow for registration to native space, a coplanar T1-weighted anatomical scan was also acquired from each participant: TE = $2.56 \mathrm{~ms}, \mathrm{TR}=1.69 \mathrm{~s}, 1 \mathrm{~mm}^{3}$ voxel size, flip angle $=12^{\circ}$.

\section{MRI Data Preprocessing}

The Oxford Center for Functional MRI of the Brain's Software Library (FSL version 6.0) was used to conduct all preprocessing and analyses on MRI data (Smith et al., 2004; Woolrich et al., 2009). Reconstructed functional volumes underwent head motion correction to the median functional volume. Non-brain tissue was removed from all functional and structural volumes and after a series of data quality checks, functional volumes underwent interleaved slice-timing correction, pre-whitening, spatial smoothing (using a $6 \mathrm{~mm}$ full-width-half-maximum Gaussian kernel), and temporal high-pass filtering (100s cutoff). These processed brain volumes were then fed into subsequent data analyses.

\section{MRI Analyses}

Preprocessed fMRI data from the Cyberball rejection task was analyzed using a two-level general linear modeling approach.

First-level (within participants). Each participant's whole-brain functional volumes were entered into a fixed-effects analysis that modeled trials as events using a canonical double-gamma hemodynamic response function with a temporal derivative. 
Regressors for Cyberball Task included Acceptance and Rejection blocks while leaving 'Rest' trials un-modeled to create an implicit baseline. 'Get Ready' trials were modeled as a nuisance regressor. All six head motion parameters from each participant were modeled as nuisance regressors for each task. Linear contrasts compared rejection to acceptance (Reject > Accept) and each condition to the implicit baseline (Accept > Baseline, Reject > Baseline). Participants' whole-brain contrast maps from these analyses were first linearly registered to their own native space structural volumes and then spatially normalized to a Montreal Neurological Institute (MNI) stereotaxic space template image, resampled into $2 \mathrm{~mm}^{3}$ voxels.

Second-level (across participants). Each participant's spatially-normalized contrast volumes from the first level were then fed into mixed effects GLMs that created group average whole-brain maps for each contrast. Cluster-based thresholding was applied to each of the group activation maps (Heller, Stanley, Yekutieli, Rubin, \& Benjamini, 2006; Worsley, 2001). Clusters were initially identified by applying a $Z>2.3$ cluster formation threshold to each group activation map. Family-wise error correction was then applied to each cluster based on Gaussian random field theory (corrected $p<$ .05). Group-level analyses proceeded in three phases. First, group contrast maps were exclusively created for participants in the opioid misuse sample, excluding controls. Second, all 56 participants were combined into group contrast maps that then compared the opioid misuse sample (coded as 1) to the control sample (coded as 0; Opioid Misuse $>$ Controls). Because the opioid misuse sample was considerably older than the control sample, $t(54)=9.79, p<.001, d=2.66$, these analyses also included age as a continuous covariate. Finally, we returned to the opioid misuse sample to conduct a 
whole-brain regression analyses that tested the effect of post-rejection opioid cravings as a continuous regressor-of-interest.

\section{Results}

\section{Descriptive Statistics}

All self-report measures exhibited substantial within-person variability and internal consistency within each sample (Table 2). Within the opioid misuse sample, opioid cravings were unassociated with rejection-related threat, as measured by the total NTS, $r(25)=.12, p=.552$, as well as its subscales of belonging threat, $r(25)=-.02$, $p=.931$, control threat, $r(25)=.24, p=.230$, meaningful existence threat, $r(25)=.13, p$ $=.521$, and self-esteem threat, $r(25)=.00, p=.993$.

\section{Table 2}

Descriptive statistics for key study variables, by sample. The control sample used a 5point NTS response scale and the opioid misuse sample used a 7-point NTS response scale.

\begin{tabular}{llllll}
\hline Sample & Variable & $M$ & $S D$ & Range & $\omega$ \\
\hline Control & NTS - Total & 2.88 & 0.63 & $1.90-4.25$ & .92 \\
& NTS - Belonging Threat & 2.79 & 0.67 & $1.80-4.40$ & .67 \\
& NTS - Control Threat & 3.40 & 0.77 & $1.80-5.00$ & .87 \\
& NTS - Meaning Threat & 2.69 & 0.85 & $1.20-5.00$ & .74 \\
& NTS - Self-Esteem Threat & 2.64 & 0.80 & $1.20-4.60$ & .86 \\
Opioid Misuse & NTS - Total & 3.91 & 0.87 & $2.00-6.10$ & .86 \\
& NTS - Belonging Threat & 4.29 & 1.17 & $2.00-6.40$ & .75 \\
& NTS - Control Threat & 4.51 & 1.18 & $2.40-7.00$ & .79 \\
& NTS - Meaning Threat & 3.78 & 1.21 & $1.40-7.00$ & .80 \\
& NTS - Self-Esteem Threat & 3.04 & 1.21 & $1.40-5.40$ & .80 \\
& Opioid Cravings & 4.46 & 1.99 & $1.00-7.00$ & n/a \\
\hline
\end{tabular}

\section{Neural Correlates of Rejection Within the Opioid Misuse Sample}

Among participants who misuse opioids, social rejection (compared to social acceptance) during the Cyberball task was both positively and negatively associated with large swaths of neural activity (Figure 1; Table 3). As in previous research with controls (e.g., Chester et al., 2018), the positively-associated regions included brain 
areas previously associated with the experience of social pain (dorsal ACC, bilateral anterior insula, thalamus), the regulation of social pain (ventrolateral prefrontal cortex), and the mentalizing network (dorsomedial prefrontal cortex, posterior cingulate cortex \precuneus, right temporoparietal junction, and bilateral temporal poles).

\section{Figure 1}

Rejection-related neural activity in mentalizing and social pain networks within the sample of participants who misused opioids.

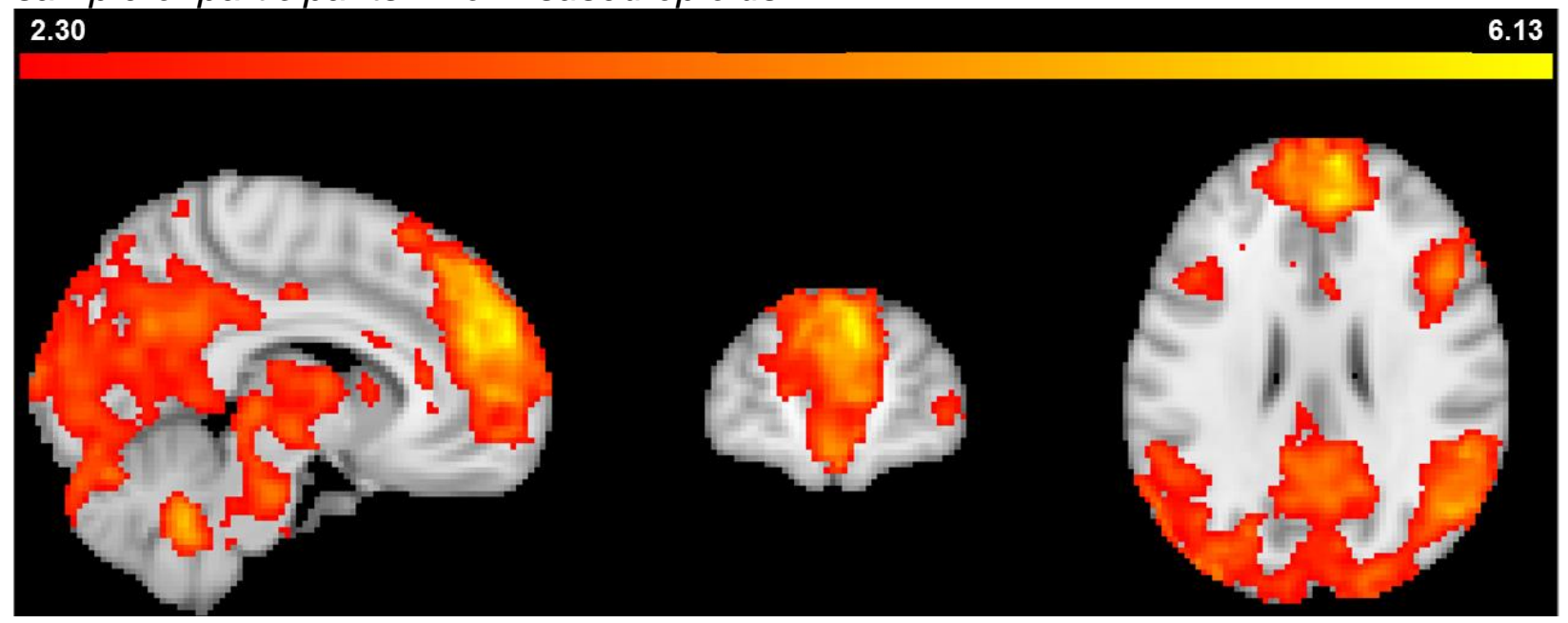

\section{Table 3}

Brain regions that were positively (cluster 1) and negatively (cluster 2) associated with Reject > Accept within the sample of participants who misused opioids. Coordinates are in MNI space.

\begin{tabular}{|c|c|c|c|c|}
\hline Cluster & Voxels & Brain Regions & Peak $Z$ & Peak $x, y, z$ \\
\hline \multirow[t]{17}{*}{1} & 57,609 & Dorsomedial prefrontal cortex & 6.13 & $8,58,26$ \\
\hline & & Cerebellum & 5.81 & $52,22,12$ \\
\hline & & Temporoparietal junction & 5.22 & $54,-42,18$ \\
\hline & & Ventrolateral prefrontal cortex & 5.10 & $-46,24,-8$ \\
\hline & & & 4.97 & $50,28,-12$ \\
\hline & & Dorsolateral prefrontal cortex & 4.98 & $44,20,30$ \\
\hline & & & 4.65 & $-28,28,42$ \\
\hline & & Temporal poles & 4.69 & $50,4,-30$ \\
\hline & & & 3.87 & $-48,10,-32$ \\
\hline & & Ventromedial prefrontal cortex & 4.62 & $-2,60,-12$ \\
\hline & & Poster cingulate cortex / Precuneus & 4.18 & $2,-52,34$ \\
\hline & & Brainstem & 4.14 & $2,-22,-24$ \\
\hline & & Anterior insula & 3.88 & $34,22,-4$ \\
\hline & & & 3.13 & $-30,16,-12$ \\
\hline & & Dorsal anterior cingulate cortex & 3.86 & $-4,26,16$ \\
\hline & & Occipital cortex & 3.78 & $-38,-86,-4$ \\
\hline & & Thalamus / Caudate & 3.31 & $8,-8,10$ \\
\hline
\end{tabular}


\begin{tabular}{ccccc}
2 & 1,941 & Postcentral / Precentral gyri & -4.52 & $-46,-24,58$ \\
\hline Associations Between Rejection-Related Brain Activity and Opioid Cravings
\end{tabular}

Among participants who misused opioids, we conducted a whole-brain regression analysis on the Reject $>$ Baseline and Accept $>$ Baseline contrasts, with mean-centered self-reported craving for opioids as the regressor-of-interest. No significant associations were observed for Reject > Baseline. However, for the Accept > Baseline contrast, opioid cravings were negatively associated with activation in the rostral ACC and VMPFC (721 voxels; peak voxel: $Z=-3.51$; MNI coordinates $x=4, y=$ $42, z=8$; Figure 2).

\section{Figure 2}

Acceptance-related neural activity in rostral ACC and VMPFC that is negatively associated with opioid cravings, within the sample of participants who misused opioids.

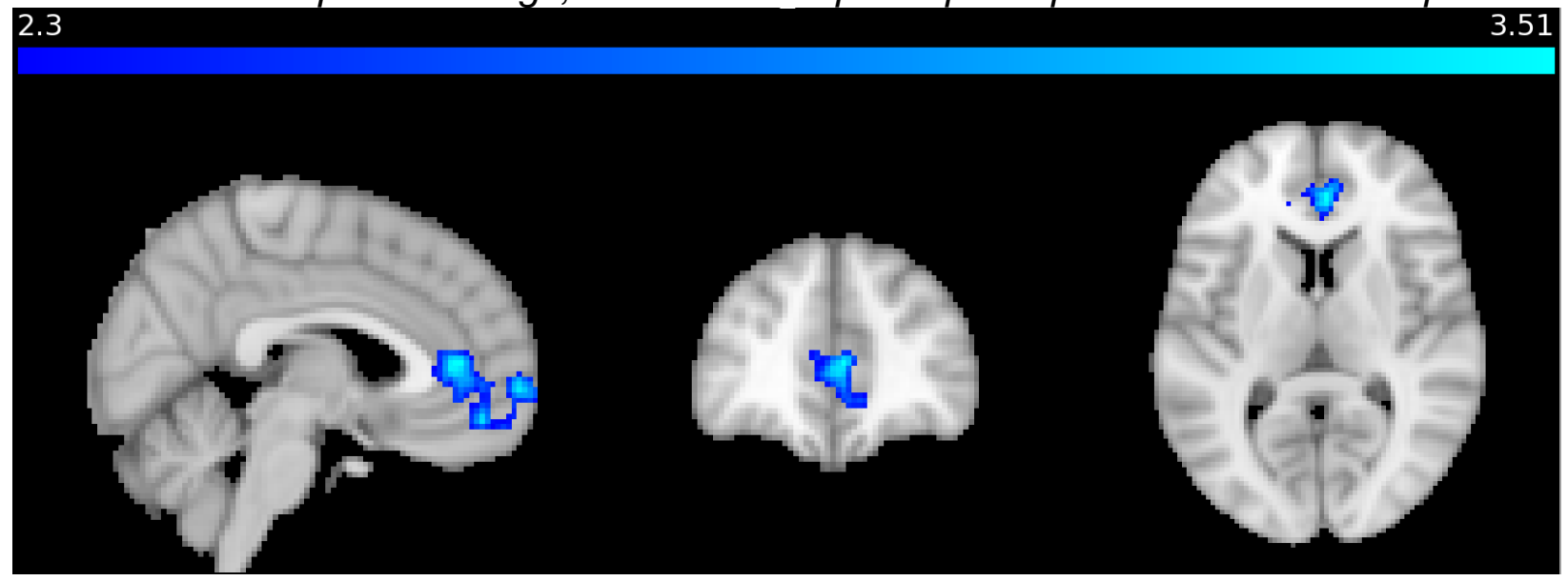

Group Differences in Neural Correlates of Rejection

After controlling for age, participants who misused opioids exhibited greater activity than controls in the ventromedial prefrontal cortex (VMPFC), subgenual ACC, and the right lateral occipital cortex (Figure 3; Table 4). 


\section{Figure 3}

Greater rejection-related neural activity among participants who misused opioids, as compared to controls, in rostral and ventral MPFC, subgenual ACC, and lateral occipital cortex.

\section{3}
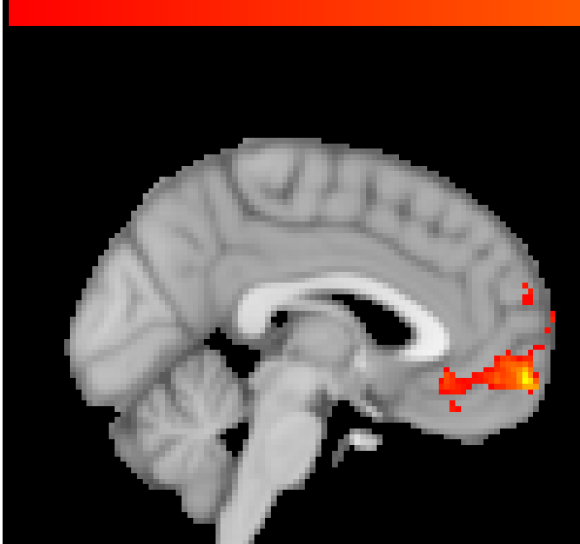

\section{Table 4}

Brain regions that exhibited greater rejection-related activity among participants who misused opioids compared to controls. Coordinates are in MNI space.

\begin{tabular}{|c|c|c|c|c|}
\hline Cluster & Voxels & Brain Regions & Peak $x, y, z$ & Peak $Z$ \\
\hline 1 & 568 & $\begin{array}{l}\text { Ventromedial prefrontal cortex / } \\
\text { Subgenual anterior cinqulate cortex }\end{array}$ & $-2,60,-10$ & 4.25 \\
\hline 2 & 908 & Lateral occipital cortex & $50,-78,-8$ & 4.24 \\
\hline
\end{tabular}

To capture the subjective distress of the rejection incident, we re-ran these group

comparisons with overall Need Threat Scale scores as a whole-brain regressor

(standardized within each sample). Compared to controls, participants who misused

opioids exhibited greater rejection-threat-related activity in one cluster that centered on

the subcallosal cortex and another cluster in the lateral occipital cortex during social

rejection (> acceptance; Figure 4; Table 5). 


\section{Figure 4}

Greater neural activity related to self-reported rejection threat among participants who misused opioids, as compared to controls, in subcallosal cortex and lateral occipital cortex.

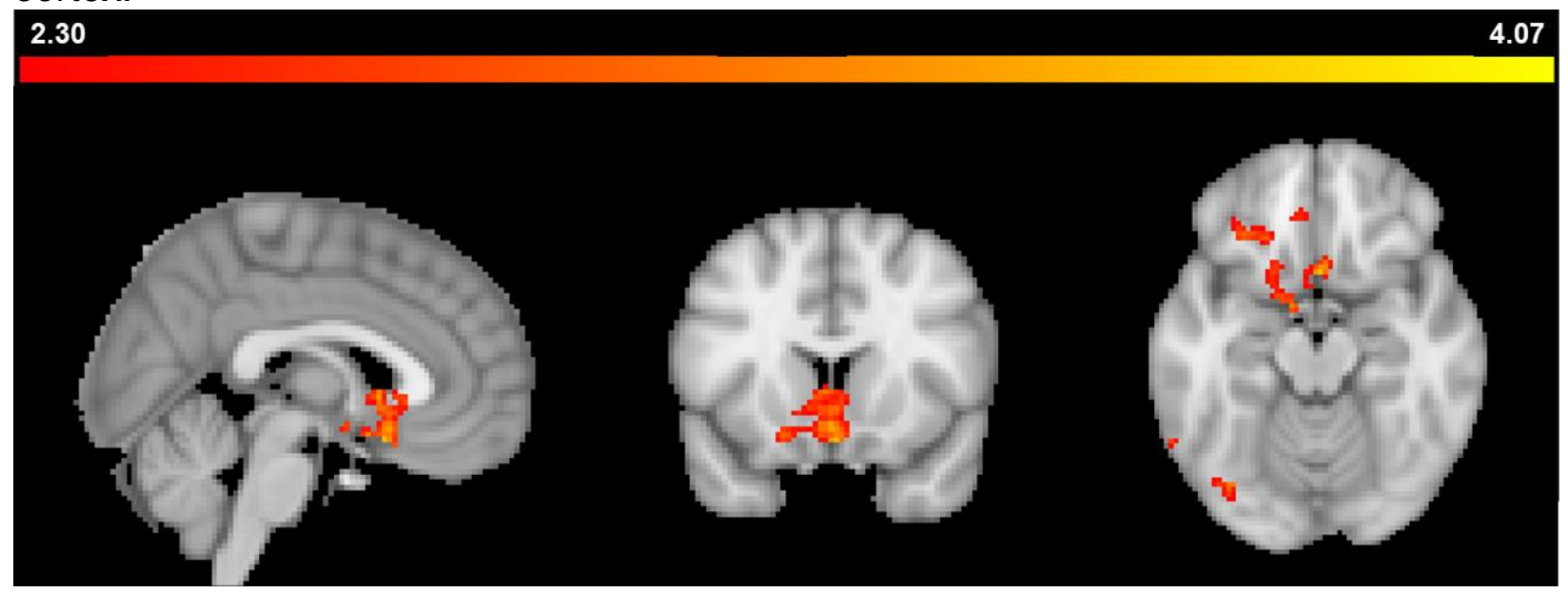

\section{Table 5}

Brain regions that exhibited greater rejection-related activity among participants who misused opioids compared to controls. Coordinates are in MNI space.

\begin{tabular}{lllll}
\hline Cluster & Voxels & Brain Regions & Peak x,y,z & Peak $Z$ \\
\hline 1 & 1,262 & Lateral occipital cortex & $-36,-98,0$ & 4.07 \\
2 & 853 & Subcallosal cortex & $2,14,-16$ & 3.86 \\
\hline
\end{tabular}

\section{Discussion}

Social rejection and its associated sting are likely contributors to drug abuse and addiction (Leach \& Kranzler, 2013; Maurage et al., 2012). However, the role of social pain and altered neural responses to social rejection in opioid misuse remain largely unknown. Using fMRI, we examined neural reactivity to social rejection among participants who recreationally misused opioids and healthy controls. We found striking similarities and differences in brain responses to rejection and neural links to opioid cravings among people who misused opioids.

\section{Neural Correlates of Rejection Within Opioid Misuse Sample}

Looking within the opioid misuse sample, we observed broad swaths of rejectionrelated brain activity that largely mirrored those observed in healthy samples. The heightened reactivity to rejection in the mentalizing and social pain networks replicates 
past work implicating these circuits as critical neural correlates of rejection (Chester et al., 2018; Eisenberger et al., 2003; Rotge et al., 2014). The large clusters of brain activity speak to the profoundly impactful nature of rejection and lend further empirical support to Social Pain Overlap Theory (Eisenberger \& Lieberman, 2004; MacDonald \& Leary, 2005). Moreover, these results suggest that, for the most part, typical neural responses to rejection are intact among people who misuse opioids.

\section{Links Between Opioid Craving and Reduced Acceptance-Related VMPFC Activity}

When we shifted our focus from rejection to acceptance, we observed that greater opioid cravings within the opioid misuse sample was linked to reduced acceptance-related VMPFC activity. In the context of socially non-threatening experiences such as acceptance, VMPFC activity reflects a 'safety signal' that suppresses perceptions of threat and inhibits social pain (Eisenberger et al., 2011; Muscatell \& Eisenberger, 2012). Therefore, opioid cravings may arise, in part, from a failure to adequately process social affiliation and inclusion in a non-threatening manner. This failure to recruit a neural safety signal may result in chronic threat processing in non-threatening social situations. Persistent experiences of social threat may then motivate cravings for opioid misuse in order to mitigate this distressing experience.

\section{Group Differences in Rejection-Related VMPFC Activity}

In contrast to the broad similarities in neural responses to rejection, we also found that participants who misused opioids exhibited greater VMPFC activity during the rejection than healthy controls. In the context of painful and threatening situations such as rejection, VMPFC activity often reflects emotion-regulation processes that suppress 
the aversive experience (Eisenberger et al., 2011; Muscatell \& Eisenberger, 2012). This VMPFC finding suggests that individuals who misuse opioids may excessively recruit the regulatory functions of the prefrontal cortex during rejection, a tendency that is reliably-linked to subsequent increased substance misuse (Chester et al., 2016). Further, we observed greater rejection-related activity in the subgenual ACC and rejection-threat-related activity in subcallosal cortex, which are both cortical regions with substantial opioid receptor density (Hsu et al., 2013). These findings suggests that opioid misuse may fundamentally alter how opioidergic cortical systems respond to social rejection, potentially causing these normally pain-reducing regions to encode greater social pain. More research should be conducted into this region's role in motivating opioid misuse after social rejection and isolating, using imaging modalities (e.g., position emission tomography) that can identify whether opioid receptor activity and binding underlie such effects

We also observed that participants who misused opioids exhibited greater rejection-related and rejection-threat-related activity in the lateral occipital cortex, which is inconsistent with studies that found blunted occipital responses to rejection in this population (Bach et al., 2019). Future work is needed to replicate these effects and examine how visual processing of socially-exclusionary events may be impacted by opioid misuse.

\section{Limitations and Future Directions}

Our investigations were limited in several key ways. First, we matched our controls on sex, but our opioid misuse sample was older and possibly differed from controls on other dimensions that could have impacted our results (e.g., socioeconomic 
status). Future research should address this issue by comparing participants who misused opioids to controls who are matched on a greater number of potentially confounding variables or by examining within-person effects of opioid misuse on social pain over time. The sub-samples were also scanned using different MRI scanners (though they were from the same manufacturer and all controllable scanning parameters were kept constant across both samples). As such, our findings (or lack thereof) could be due to inherent differences in the two scanners' MRI data acquisition and processing. Future work should compare controls to participants who misuse opioids using identical scanning equipment and parameters. Second, our findings were largely correlational and future work should experimentally manipulate social pain, emotion-regulation, opioid functioning and cravings to identify causal effects. Third, participants in the opioid misuse sample were verified to be sober at the time of testing and therefore symptoms of withdrawal may have affected our results. Covarying measures of withdrawal symptoms and administering opioid maintenance treatments in future investigations would help alleviate this issue.

\section{Conclusions}

Opioid misuse represents a public health crisis with great societal costs. We found evidence for two neural and psychological mechanisms that may subserve such addictive behavior. Indeed, our findings suggest that opioid misuse may result from (A) excessive regulatory responses in the prefrontal cortex and (B) insufficient neural signaling that social acceptance is safe and non-threatening. We are hopeful that these neural pathways are fruitful targets for future investigations into the social-affective bases of opioid misuse and interventions that seek to reduce such addictive behaviors. 


\section{Acknowledgments}

Research reported in this publication was supported by an Ignite Collaborative Research Grant from the University of Kentucky's Office of the Vice President for

Research and the National Institute on Alcohol Abuse and Alcoholism (NIAAA) of the National Institutes of Health under award number K01AA026647 (PI: Chester). 


\section{References}

Bach, P., Frischknecht, U., Bungert, M., Karl, D., Vollmert, C., Vollstädt-Klein, S., ... \& Hermann, D. (2019). Effects of social exclusion and physical pain in chronic opioid maintenance treatment: $\mathrm{fMRI}$ correlates. European Neuropsychopharmacology, 29(2), 291-305.

Bershad, A. K., Seiden, J. A., \& de Wit, H. (2016). Effects of buprenorphine on responses to social stimuli in healthy adults. Psychoneuroendocrinology, 63, 4349.

Carlyle, M., Rowley, M., Stevens, T., Karl, A., \& Morgan, C. J. (in press). Impaired empathy and increased anger following social exclusion in non-intoxicated opioid users. Psychopharmacology.

Chester, D. S. \& DeWall, C. N. (2018). Aggression is associated with greater subsequent alcohol consumption: Shared neural basis in the ventral striatum. Aggressive Behavior, 44(3), 285-293.

Chester, D. S., Eisenberger, N. I., Pond, R. S., Richman, S. B., Bushman, B. J., \& DeWall, C. N. (2014). The interactive effect of social pain and executive functioning on aggression: An fMRI experiment. Social Cognitive and Affective Neuroscience, 9(5), 699-704.

Chester, D. S., Lynam, D. R., Milich, R., \& DeWall, C. N. (2018). Neural mechanisms of the rejection-aggression link. Social Cognitive and Affective Neuroscience, 13(5), 501-512.

Chester, D. S., Lynam, D. R., Milich, R., Powell, D. K., Andersen, A. H., \& DeWall, C. N. (2016). How do negative emotions impair self-control? A neural model of 
negative urgency. Neurolmage, 132, 43-50.

Contet, C., Kieffer, B. L., \& Befort, K. (2004). Mu opioid receptor: a gateway to drug addiction. Current Opinion in Neurobiology, 14(3), 370-378.

DeWall, C. N., MacDonald, G., Webster, G. D., Masten, C. L., Baumeister, R. F., Powell, C., ... \& Eisenberger, N. I. (2010). Acetaminophen reduces social pain: Behavioral and neural evidence. Psychological Science, 21(7), 931-937.

Eisenberger, N. I. (2012). The pain of social disconnection: Examining the shared neural underpinnings of physical and social pain. Nature Reviews Neuroscience, 13(6), 421-434.

Eisenberger, N. I., Jarcho, J. M., Lieberman, M. D., \& Naliboff, B. D. (2006). An experimental study of shared sensitivity to physical pain and social rejection. Pain, 126(1-3), 132-138.

Eisenberger, N. I., \& Lieberman, M. D. (2004). Why rejection hurts: A common neural alarm system for physical and social pain. Trends in Cognitive Sciences, 8(7), 294-300.

Eisenberger, N. I., Lieberman, M. D., \& Williams, K. D. (2003). Does rejection hurt? An fMRI study of social exclusion. Science, 302(5643), 290-292.

Eisenberger, N. I., Master, S. L., Inagaki, T. K., Taylor, S. E., Shirinyan, D., Lieberman, M. D., \& Naliboff, B. D. (2011). Attachment figures activate a safety signal-related neural region and reduce pain experience. Proceedings of the National Academy of Sciences, 108(28), 11721-11726.

Garland, E. L., Bryan, C. J., Nakamura, Y., Froeliger, B., \& Howard, M. O. (2017). Deficits in autonomic indices of emotion regulation and reward processing 
associated with prescription opioid use and misuse.

Psychopharmacology, 234(4), 621-629

Heller, R., Stanley, D., Yekutieli, D., Rubin, N., \& Benjamini, Y. (2006). Cluster-based analysis of fMRI data. Neurolmage, 33(2), 599-608.

Hsu, D. T., Sanford, B. J., Meyers, K. K., Love, T. M., Crocker, J. K., Langenecker, S. A., \& Zubieta, J. K. (2013). Response of the $\mu$-opioid system to social rejection and acceptance. Molecular Psychiatry, 18(11), 1211-1217.

Hsu, D. T., Sanford, B. J., Meyers, K. K., Love, T. M., Hazlett, K. E., Walker, S. J., ... \& Zubieta, J. K. (2015). It still hurts: altered endogenous opioid activity in the brain during social rejection and acceptance in major depressive disorder. Molecular Psychiatry, 20(2), 193-201.

Inagaki, T. K. (2018). Opioids and social connection. Current Directions in Psychological Science, 27(2), 85-90.

Inagaki, T., Irwin, M., \& Eisenberger, N. (2015). Blocking opioids attenuates physical warmth-induced feelings of social connection. Emotion, 15(4), 494-500.

Inagaki, T. K., Ray, L. A., Irwin, M. R., Way, B. M., \& Eisenberger, N. I. (2016). Opioids and social bonding: Naltrexone reduces feelings of social connection. Social Cognitive and Affective Neuroscience, 11(5), 728-735.

Kroll, S. L., Williams, D. P., Thoma, M., Staib, M., Binz, T. M., Baumgartner, M. R., ... \& Quednow, B. B. (2019). Non-medical prescription opioid users exhibit dysfunctional physiological stress responses to social rejection. Psychoneuroendocrinology, 100, 264-275.

Leach, D. \& Kranzler, H. R. (2013). An interpersonal model of addiction 
relapse. Addictive Disorders \& Their Treatment, 12(4), 183-192.

MacDonald, G. \& Leary, M. R. (2005). Why does social exclusion hurt? The relationship between social and physical pain. Psychological Bulletin, 131(2), 202-223.

Maurage, P., Joassin, F., Philippot, P., Heeren, A., Vermeulen, N., Mahau, P., ... \& Luminet, O. (2012). Disrupted regulation of social exclusion in alcoholdependence: an fMRI study. Neuropsychopharmacology, 37(9), 2067-2075.

Muscatell, K. A., \& Eisenberger, N. I. (2012). A social neuroscience perspective on stress and health. Social and Personality Psychology Compass, 6(12), 890-904.

National Survey on Drug Use and Health. (2019). Key substance use and mental health indicators in the United States: Results from the 2018 National Survey on Drug Use and Health. Retrieved from http://www.samhsa.gov/data/data-wecollect/nsduh-national-survey-drug-use-and-health.

Nummenmaa, L. \& Tuominen, L. (2018). Opioid system and human emotions. British Journal of Pharmacology, 175(14), 2737-2749.

Panksepp, J., Herman, B., Conner, R., Bishop, P., \& Scott, J. P. (1978). The biology of social attachments: opiates alleviate separation distress. Biological psychiatry, 13(5), 607-618.

Riva, P., Wirth, J. H., \& Williams, K. D. (2011). The consequences of pain: The social and physical pain overlap on psychological responses. European Journal of Social Psychology, 41, 681-687.

Rotge, J. Y., Lemogne, C., Hinfray, S., Huguet, P., Grynszpan, O., Tartour, E., ... \& Fossati, P. (2014). A meta-analysis of the anterior cingulate contribution to social pain. Social Cognitive and Affective Neuroscience, 10(1), 19-27. 
Smith, S. M., Jenkinson, M., Woolrich, M. W., Beckmann, C. F., Behrens, T. E. J., Johansen-Berg, H., ... Matthews, P. M. (2004). Advances in functional and structural MR image analysis and implementation as FSL. Neurolmage, 23(1), S208-S219.

Van Beest, I., \& Williams, K. D. (2006). When inclusion costs and ostracism pays, ostracism still hurts. Journal of Personality and Social Psychology, 91, 918-928.

Voon, P., Karamouzian, M., \& Kerr, T. (2017). Chronic pain and opioid misuse: A review of reviews. Substance Abuse Treatment, Prevention, and Policy, 12(1), 36.

Williams, K. D. (2009). Ostracism: A temporal need-threat model. In Mark P. Zanna (Ed.), Advances in experimental social psychology (pp. 275-314). Cambridge, MA: Academic Press.

Williams, K. D., Cheung, C. T, K., \& Choi, W. (2000). Cyberostracism: Effects of being ignored over the Internet. Journal of Personality and Social Psychology, 79(5), 748-762.

Woolrich, M. W., Jbabdi, S., Patenaude, B., Chappell, M., Makni, S., Behrens, T., ... Smith, S. M. (2009). Bayesian analysis of neuroimaging data in FSL. Neurolmage, 45(1), S173-S186.

Worsley, K. J. (2001). Statistical analysis of activation images. Functional MRI: An Introduction to Methods, 14(1), 251-270. 